\title{
Galactose Induces Formation of Chains of the Retinal Adhesion Protein, Retinoschisin
}

J. Bernard Heymann ${ }^{1}$, Camasamudram Vijayasarathy ${ }^{2}$, Rick Huang ${ }^{3}$, Altaira Dearborn ${ }^{1}$, Paul A. Sieving ${ }^{2,4}$ and Alasdair C. Steven ${ }^{1}$

${ }^{1 .}$ Laboratory for Structural Biology Research, NIAMS, NIH, Bethesda, MD.

2. Section on Translational Research for Retinal and Macular Degeneration, NIDCD, NIH, Bethesda, MD.

${ }^{3 .}$ Cryo-Electron Microscopy Facility, Janelia Research Campus, HHMI, Ashburn, VA.

4. NEI, NIH, Bethesda, MD.

Retinoschisin (RS1) is involved in cell-cell junctions in the retina, but is unique among known celladhesion proteins in that it is a soluble secreted protein. Loss-of-function mutations in RS1 lead to early vision impairment in young males, called X-linked retinoschisis (XLRS). This name refers to the separation of the inner retinal layers, disrupting synaptic signaling. We previously reported the structure to $4.1 \AA$, revealing double octamer rings [1]. Each $24 \mathrm{kDa}$ subunits has a small N-terminal RS1 domain in the middle of the ring, and a larger C-terminal discoidin domain at the periphery. As a soluble protein, RS1 is unlikely to interact directly with the hydrophobic parts of the cell membrane. Importantly, RS1 binds several sugar moieties, of which the best characterized is galactose [2]. It may tie retinal membranes together through interaction with the abundant glycans found on lipid-embedded and extracellular matrix proteins.

We imaged RS1 (purified as in [1]) in the presence of galactose by cryo-electron microscopy. Surprisingly, the RS1 double octamer rings formed strings of molecules (Figure 1A) not seen without galactose. They aligned at the air-water interface to show mostly one or a small number of side views. We processed these (using the Bsoft package [3]) to find classes for averaging and interpretation: Figure 1B shows the six best class averages. The repeat along the chain is $124 \AA$, somewhat less than the maximum diameter of $140 \AA$ (measured between the subunit spike tips). Curiously, the central planes through the rings from one molecule to the next do not line up, but are offset by $\sim 13 \AA$. Along a chain of molecules, the offset switches direction at every interface, giving a slightly zig-zag appearance. This indicates that the interaction propagates along the chain, with some communication of the conformation through the rings. We determined the orientation of each double ring to within a small uncertainty $\left( \pm 3^{\circ}\right)$, allowing us to place 3D maps in the correct relationship with each other (Figure 2A). The interaction between the double rings is through the spikes, with contributions from four subunits of each double ring (Figure 2B). The interface between neighboring double rings appears to be extensive and well-formed (Figure 2C). However, the two rings must break their symmetry to produce the alternating offset, likely with a different interface from that portrayed in Figure 2C. Future studies will focus on obtaining better 3D maps of the chains, locating galactose in the map, investigating the binding of other carbohydrates and assessing the implications of these interactions in the retina [4].

\section{References:}

[1] G Tolun et al, PNAS 113 (2016), p. 5287.

[2] FM Dyka et al, Biochemistry 47 (2008), p. 9098.

[3] JB Heymann and DM Belnap, J Struct Biol 157 (2007), p. 3. 
[4] This work was supported by the Intramural Research Programs of NEI, NIDCD and NIAMS.
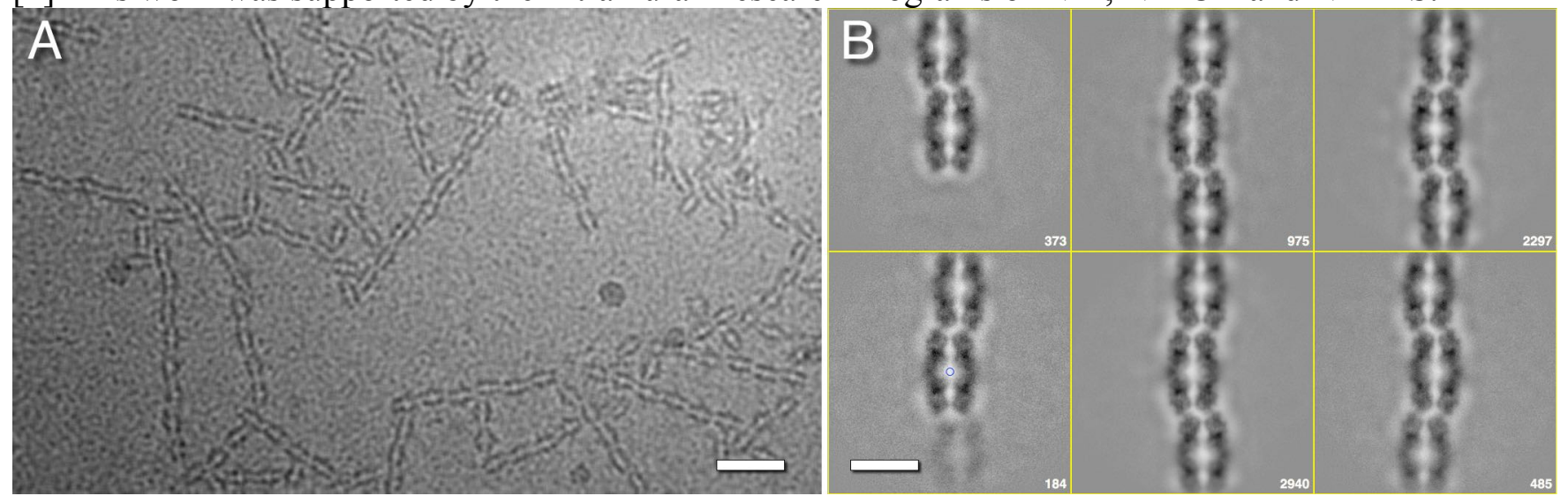

Figure 1. (A) Cryo-electron micrograph of retinoschisin $(\sim 0.14 \mathrm{mg} / \mathrm{ml})$ with galactose $(10 \mathrm{mM})$ taken on a FEI Krios with a Gatan K2 camera in super-resolution mode (dose of $4 \mathrm{e}^{-} / \AA^{2}$ each $0.25 \mathrm{~s}$ frame for 20 frames $=80 \mathrm{e}^{-} / \AA^{2}$ ). Scale bar: $300 \AA$. (B) Class averages of chains of RS1 double rings (with the number of contributing images), showing a lateral offset propagated in alternating fashion along the chain. Scale bar: $100 \AA \AA$.
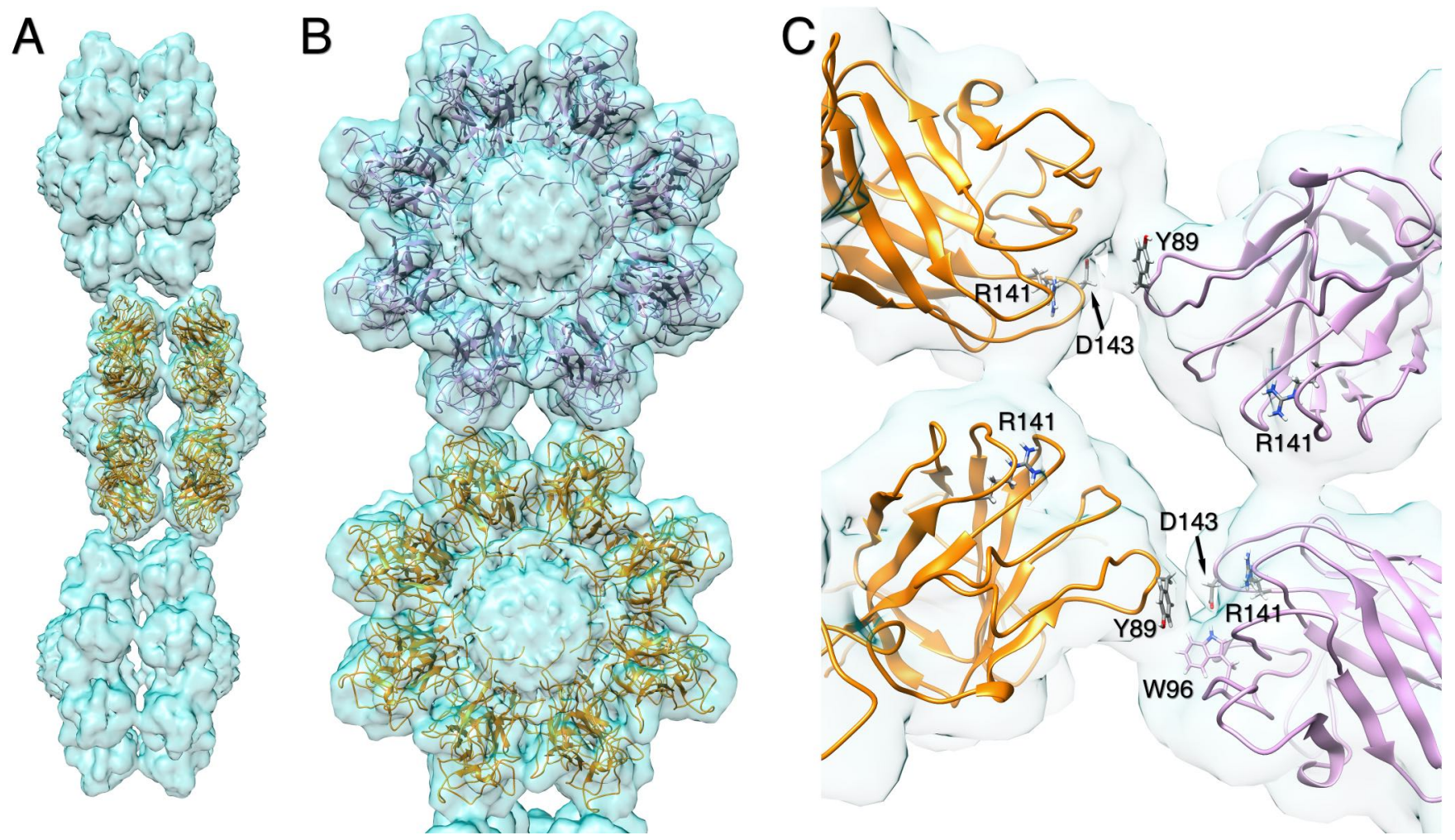

Figure 2. Assembly of 3D maps and models consistent with the major view of the double rings in the chains. (A) Side view as seen in the micrographs. (B) Top view, showing the interaction between the subunits of the double rings. (C) The interface between the double rings shows good connectivity and little overlap, despite being refined on isolated double rings in a previous study [1]. Two different interface types connect the two pairs of rings. The important R141 is buried in the subunit core and may not directly contribute to the interface. A few other residues that lead to disease when mutated are noted. Map source: EMDB 6425; Atomic structure source: PDB 3JD6. 